\section{Unverzichtbar nicht nur für Kinderärzte}

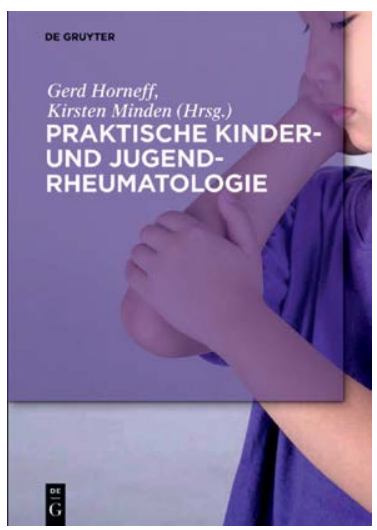

Horneff G, Minden K. Praktische Kinder- und Jugendrheumatologie. Berlin/Boston: Verlag Walter de Gruyter GmbH; 2020. 69,95€. ISBN: 978-3-11-049630-7
Die rheumatologische Fachliteratur hat sehr erfreulichen Zuwachs bekommen. Im September 2020 erschien das Lehrbuch „Praktische Kinder- und Jugendrheumatologie“, herausgegeben von Herrn Prof. Gerd Horneff und Frau Prof. Kirsten Minden. An diesem Buch haben 17 namhafte Kinderrheumatologen, überwiegend aus Deutschland, aber auch aus England mitgewirkt. Es bietet einen hervorragenden Überblick über sämtliche rheumatologische Erkrankungen des Kindesalters, aber auch über kinderrheumatologisch relevante Differenzialdiagnosen, wie genetische Störungen des Bindegewebsstoffwechsels oder onkologische Krankheitsbilder. Das Werk richtet sich in erster Linie an praktisch tätige ÄrztInnen und verzichtet daher auf eine umfangreiche Darstellung immunologischer Grundlagen oder ätiologischer Zusammenhänge.

Dem Anspruch, für die tägliche Praxis hilfreich zu sein, wird das Buch vollauf gerecht. Im ersten, den Grundlagen der Kinderrheumatologie gewidmeten Teil des Buches wird ausführlich und instruktiv auf Untersuchung und Anameseerhebung eingegangen. Die Besonderheiten der Untersuchungstechnik bei Kindern und das Denken in Leitsymptomen wie Gelenkschwellungen oder Rückenschmerz erfahren erfreuliche Berücksichtigung. In den Abschnitten zur Labordiagnostik finden sich neben der tabellarischen Abhandlung einzelner Parameter auch schlüssige Vorschläge zu Laborprogrammen, die bei dem Verdacht auf eine entzündliche Gelenkerkrankung, eine Kollagenose oder eine Vaskulitis hilfreich sein können. Etwas fragmentarisch ist die radiologische Bildgebung geraten. In der konventionellen Röntgendiagnostik findet sich lediglich der Hinweis darauf, dass die Strahlenbelastung „zu berücksichtigen“ sei. Konkrete Angaben zur Strahlendosis einzelner Untersuchungen wären für den Alltag dienlich gewesen.

Der erste Teil des Buches enthält außerdem Grundlegendes zur medikamentösen und nichtmedikamentösen Therapie. Hier werden von nichtsteroidalen Antirheumatika bis zu Biologika und JAK-Inhibitoren sämtliche relevanten Arzneimittelgruppen der Kinderrheumatologie behandelt.
Das Buch ist durch zahlreiche hochauflösende klinische Bilder und durch sinnvolle Schemadarstellungen illustriert. Eine Vielzahl von Tabellen ordnet Symptome, Differenzialdiagnosen und Klassifikationskriterien sinnvoll. Jedes Kapitel ist mit einem ausführlichen Literaturteil zum vertiefenden Studium versehen.

Lobenswert ist auch das ausführliche Eingehen auf nichtentzündliche Schmerzsyndrome und ihre auf psychopathologische Krankheitsbilder hinweisende Differenzialdiagnostik. Sehr wertvoll ist der ausführliche und systematisch gegliederte Abschnitt zu den autoinflammatorischen Syndromen, der Kinderrheumatologen und Kinderärzten Einblick in dieses auf den ersten Blick verwirrende und vielfältige Themengebiet liefert.

Zusammenfassend füllt dieses Fachbuch eine wichtige Lücke in der rheumatologischen Fachliteratur. Auch wenn die Herausgeber in maßvollem Understatement im Vorwort äußern, dass ihr Buch nicht den Anspruch habe, Lehr- oder Handbuch zu sein, bietet es eine sehr umfassende Vielfalt nützlicher Informationen. Das Werk ist daher überaus geeignet, Kinderrheumatologen, internistischen Rheumatologen, Kinderärzten, aber auch Orthopäden und Hausärzten Hilfe und Unterstützung bei der Behandlung pädiatrisch-rheumatologischer Krankheitsbilder zu bieten.

Prof. Gernot Keyßer, Halle 